\title{
He I temperatures in planetary nebulae
}

\author{
Antonio Peimbert and Manuel Peimbert \\ Instituto de Astronomía, Universidad Nacional Autónoma de México, \\ Apartado postal 70-264, México D. F. 4510, México \\ email: antonio@astroscu.unam.mx; peimbert@astroscu.unam.mx
}

\begin{abstract}
It is well established that PNe are not thermally homogeneous, beyond what chemically homogeneous photoionization models predict. To discriminate between the different scenarios proposed in the literature, we have determined the temperature based on He I lines for a dozen PNe. We compare these determinations with those derived from the Balmer continua. We find that the He I temperatures agree with the Balmer temperatures and that both are lower than those derived from CELs; suggesting that most PNe are chemically homogeneous.
\end{abstract}

Keywords. planetary nebulae: general, ISM: abundances, stars: abundances

The aim of this work is to find out if the physical conditions prevailing in the regions where the H I, He I, and O II lines originate are the same. If this is the case then the relative abundances derived from these lines will be representative of the observed regions. To obtain this aim we will compare the Balmer temperature, $T_{e}\left(\mathrm{H}^{+}\right)$, with the temperature derived from a set of $\mathrm{He}$ I recombination lines, $T_{e}\left(\mathrm{He}^{+}\right)$.

We have developed a maximum likelihood method to derive the $T_{e}\left(\mathrm{He}^{+}\right)$of a given nebula based on He I recombination lines. We require a minimum of six He I lines to derive reliable $T_{e}\left(\mathrm{He}^{+}\right)$values. This method has been applied before to derive the primordial helium abundance (see Peimbert et al. 2000 and 2007, for further details).

By comparing the temperature derived from [O III] lines, $T_{e}[\mathrm{O} \mathrm{III}]$, with $T_{e}\left(\mathrm{H}^{+}\right)$it is found that the diference between $T_{e}[\mathrm{O} \mathrm{III}]$ and $T_{e}\left(\mathrm{H}^{+}\right)$is considerably larger than predicted by photoionization models. There are two possibilities to model these temperature inhomogeneities: a) a chemical homogeneous medium, and b) a chemically inhomogeneous medium.

It is possible to discriminate between these two scenarios by comparing $T_{e}\left(\mathrm{He}^{+}\right)$with $T_{e}\left(\mathrm{H}^{+}\right)$. If $T_{e}\left(\mathrm{He}^{+}\right)<T_{e}\left(\mathrm{H}^{+}\right)$it would indicate the presence of hydrogen poor knots (oxygen rich knots). On the other hand, if $T_{e}\left(\mathrm{He}^{+}\right)=T_{e}\left(\mathrm{H}^{+}\right)$it would indicate a chemically homogeneous medium; this would imply that the thermal inhomogeneities are due to inhomogeneities in the heating rather than the cooling; and would require different conditions than those predicted by standard photoionization models.

1. Based on a maximum likelihood method applied to sets of 6 to 16 He I recombination lines for a given PN we have determined the He I electron temperature for $13 \mathrm{PNe}$ of different types.

2. For our sample of $\mathrm{PNe}$ we find that the Balmer temperature, $T_{e}\left(\mathrm{H}^{+}\right)$, is similar to the temperature derived from the He I recombination lines, $T_{e}\left(\mathrm{He}^{+}\right)$. This result implies that the physical conditions where the $\mathrm{H} \mathrm{I}$ and He I lines originate are similar.

3 . We have also compared the $T_{e}\left(\mathrm{H}^{+}\right)-T_{e}\left(\mathrm{He}^{+}\right)$values with the abundance discrepancy factors, $\mathrm{ADFs}$, for the objects in our sample and there is no correlation.

4. We compare the $\mathrm{O}(\mathrm{RL})$ and He abundances by mass of our PNe sample with: Galactic chemical evolution models (Carigi \& Peimbert 2011), the protosolar abundances (Asplund et al. 2009), and the abundances of the Galactic H II region M17 (Carigi \& Peimbert 2008). From these comparisons we find that: 


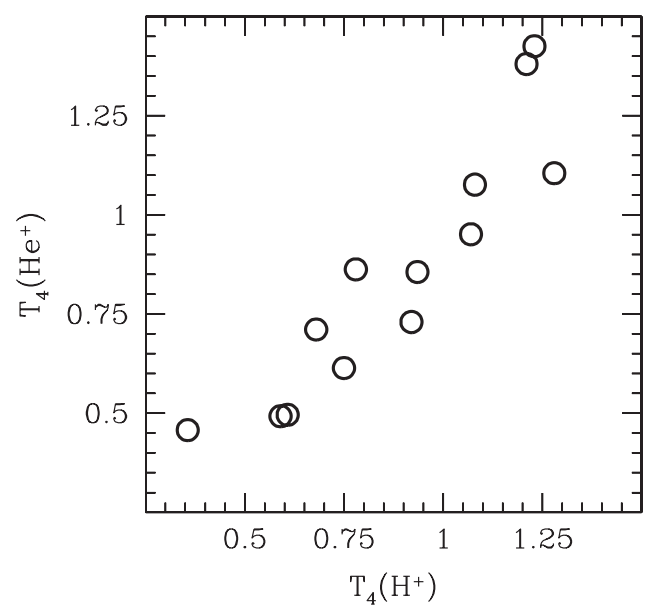

Figure 1. $T_{e}\left(\mathrm{H}^{+}\right)$obtained from the literature (Georgiev et al. 2005; Liu et al. 2000, 2001; Peimbert et al. 2004; Ruiz et al. 2003; Tsamis et al. 2003; Zhang et al. 2005) versus $T_{e}\left(\mathrm{He}^{+}\right)$ derived in this work for the following PNe: NGC 3132, NGC 3918, NGC 5307, NGC 5315, NGC 5882, NGC 6153, NGC 6543, NGC 6818, NGC 7027, IC 4191, IC 4406, M1-42, and M2-36. In this figure the typical errors for $T_{e}\left(\mathrm{He}^{+}\right)$amount to $500 \mathrm{~K}$ and for $T_{e}\left(\mathrm{H}^{+}\right)$to $750 \mathrm{~K}$, the correlation between $T_{e}\left(\mathrm{H}^{+}\right)$and $T_{e}\left(\mathrm{He}^{+}\right)$is very strong and the difference for a given object is very small supporting the idea that these objects are chemically homogeneous.

a) Disregarding M1-42, M2-36, and NGC 6153, the average $12+\mathrm{O} / \mathrm{H}$ value of the sample is $9.01,0.28$ dex higher than the protosolar value and 0.14 dex higher than the M17 value. These results imply that these objects seem to have produced some helium and some oxygen during their evolution.

b) The excess in the O abundance by mass of M1-42, M2-36 and NGC 6153 relative to the average of the other group of $\mathrm{PNe}$ is of 0.69 dex while the He abundance by mass is similar. This result implies that the $\mathrm{O}$ excess is qualitatively different to the $\mathrm{O}$ excesses of the other objects of the sample.

\section{References}

Asplund, M., Grevesse, N., Sauval, A. J., \& Scott, P. 2009, ARAA, 47, 481

Carigi, L. \& Peimbert, M. 2008, RevMexAA, 44, 311

Carigi, L. \& Peimbert, M. 2011, RevMexAA, 47, 139

Georgiev, L. N., Peimbert, M., Hillier, D. J., Richer, M. G., Arrieta, A., \& Peimbert, A. 2008, ApJ, 681, 333

Liu, X. W., Luo, S. G., Barlow, M. J., Danziger, I. J., \& Storey, P. J. 2001, MNRAS, 327, 141

Liu, X. W., Storey, P. J., Barlow, M. J., Danziger, I. J., Cohen, M., \& Bryce, M. 2000, MNRAS, 312,585

Peimbert, M., Luridiana, V., \& Peimbert, A. 2007, ApJ, 666, 636

Peimbert, M., Peimbert, A., \& Ruiz, M. T. 2000, ApJ, 541, 688

Peimbert, M., Peimbert, A., Ruiz, M. T., \& Esteban, C. 2004, ApJ, 150, 431

Ruiz, M. T., Peimbert, A., Peimbert, M., \& Esteban, C. 2003, ApJ, 595, 247

Tsamis, Y. G., Barlow, M. J., Liu, X. W., Danziger, I. J., \& Storey, P. J. 2003, MNRAS, 345, 186

Zhang, Y., Liu, X. W., Luo, S. G., Péquignot, D., \& Barlow, M. J. 2005, A $\& A, ~ 442,249$ 